\title{
Upregulation of miR-215 attenuates propofol-induced apoptosis and oxidative stress in developing neurons by targeting LATS2
}

\author{
Fang Tang, Lili Zhao, Qi Yu, Tianyin Liu, Hongyan Gong, Zhiyi Liu* (i) and Qing Li
}

\begin{abstract}
Background: Propofol is an intravenous anesthetic agent that commonly induces significant neuroapoptosis. MicroRNAs (miRNAs) have been reported to participate in the regulation of propofol exposure-mediated neurotoxicity. MiR-215, as one of miRNAs, was found to regulate nerve cell survival. However, the mechanism through which miRNAs regulate propofol exposure-mediated neurotoxicity is still unclear.

Methods: Real-time PCR was used to detect miR-215 expression level. Cell viability was measured using MTT assay. Cell apoptosis was examined via flow cytometry analysis. ROS, MDA, LDH and SOD levels were assayed through ELISA kits. Dual luciferase reporter assay identified the interaction between miR-215 and large tumor suppressor 2 (LATS2). Protein level was detected using western blot analysis.

Results: MiR-215 expression was downregulated in propofol-treated rat hippocampal neurons. MiR-215 mimics promoted cell viability and reduced apoptosis in propofol-treated neonatal rat hippocampal neuron. MiR-215 mimics also caused inhibition of oxidative stress as evidenced by suppression of ROS, MDA and LDH levels as well as increase of SOD level. In addition, we found that large tumor suppressor 2 (LATS2) is a target of miR-215 and miR-215 mimics decreased LATS2 level in propofol-treated neonatal rat hippocampal neuron. Further, LATS2 overexpression suppressed the effect of miR-215 on propofol-induced apoptosis and oxidative stress in neonatal rat hippocampal neuron.

Conclusion: Taken together, we demonstrate that miR-215 attenuates propofol-induced apoptosis and oxidative stress in neonatal rat hippocampal neuron by targeting LATS2, suggesting that miR-215 may provide a new candidate for the treatment of propofol exposure-induced neurotoxicity.
\end{abstract}

Keywords: miR-215, LATS2, Propofol, Apoptosis, Oxidative stress

\section{Background}

Propofol is an intravenous anesthetic agent commonly used for the induction and maintenance of anesthesia and sedation (Chidambaran et al. 2015). Propofol can lead to significant neuroapoptosis and affect dendrite development and cognitive function, which causes concern

\footnotetext{
* Correspondence: ZhiyiLiufjk@163.com

Department of Anesthesiology, The First Affiliated Hospital of Nanchang University, No. 17 Yongwaizheng Street, Nanchang City 330006, Jiangxi Province, China
}

about its safety in pediatric anesthesia (Bosnjak et al. 2016). Reported mechanisms of propofol exposureinduced neurotoxicity include calcium dysregulation, mitochondrial fission, abnormal expression of neurotrophic protein and neuroinflammation (Wei 2011; Cui et al. 2011; Unoki and Nakamura 2001). Therefore, it is necessary to explore the biomarker to prevent and improve propofol exposure-induced neurotoxicity.

MicroRNAs (miRNAs), 18-25 nucleotides in length, are endogenous non-coding RNA molecules that

(c) The Author(s). 2020 Open Access This article is licensed under a Creative Commons Attribution 4.0 International License, which permits use, sharing, adaptation, distribution and reproduction in any medium or format, as long as you give appropriate credit to the original author(s) and the source, provide a link to the Creative Commons licence, and indicate if changes were made. The images or other third party material in this article are included in the article's Creative Commons licence, unless indicated otherwise in a credit line to the material. If material is not included in the article's Creative Commons licence and your intended use is not permitted by statutory regulation or exceeds the permitted use, you will need to obtain permission directly from the copyright holder. To view a copy of this licence, visit http://creativecommons.org/licenses/by/4.0/. 
regulate biological processes through suppression of target messenger RNA expression (Fabian et al. 2010; Bartel 2009; Shukla et al. 2011; Ji et al. 2019). Increasing evidences have shown that miRNAs are involved in the regulation of propofol exposure-mediated neurotoxicity. For example, Jiang et al. discovered that miR-141-3p is useful for propofol-mediated suppression of neural stem cells neurogenesis (Jiang et al. 2017). Zheng et al. proved that propofol attenuates neuroinflammatory response of microglia in response to LPS via regulating miR-155 (Zheng et al. 2018). Besides, Zhang et al. verified that propofol anesthesia decreases miR-132 level and inhibits the number of dendritic spines in the hippocampus (Zhang et al. 2017). Interestingly, miR-215 is reduced in ischemic stroke, which leads to suppression of nerve cell apoptosis, autophagy, ischemic infarction and improved neurological deficit via down-regulation of nuclear factor- $\mathrm{kB}$ activator $1 /$ interleukin-17 receptor A pathway. These results suggest that miR-215 plays a neuroprotective role in ischemic injury (Sun et al. 2018). However, the role of miR-215 in propofol exposure-induced neurotoxicity is still unclear.

Large tumor suppressor 2 (LATS2) gene maps onto the human chromosome 13q11-12 (Yabuta et al. 2000). Nuclear LATS2 has been found to activate p53, maintaining the proper chromosome number when mitotic apparatus are impaired (Aylon et al. 2006). Previously, LATS2 was found to promote p53mediated apoptosis (Aylon et al. 2010) and reduce the expression of $\mathrm{BCL}-2$ and $\mathrm{BCL}-\mathrm{x}(\mathrm{L})$ (Ke et al. 2004). Moreover, Brandt et al. found that LATS2 is involved in the production of peripheral nerve sheath tumors (Brandt et al. 2019). LATS2 kinase activation inhibits Yap protein to suppress proliferation and cell cycle exit in the process of neurogenesis (Zhang et al. 2012). Notably, LATS2 is predicted to be a target of miR-215 using bioinformatic analysis. Thus, these findings suggest that miR-215 may be involved in regulation of propofol exposure-induced neurotoxicity through LATS2.

In the current study, we investigated miR-215 expression in propofol-treated rat hippocampal neurons. The effects of miR-215 on cell viability and apoptosis were then examined. Furthermore, we explored whether miR-215 modulates propofol exposure-induced neurotoxicity via LATS2. Together these results suggest a new target for the treatment of propofol exposure-induced neurotoxicity.

\section{Methods}

\section{Neonatal rat hippocampal neuron isolation, culture and} transfection

The cell isolation procedures in this study were approved by Guide for the Care and Use of Laboratory Animals and in agreement with the Ethics Committee of The first affiliated hospital of Nanchang University. Neonatal rat hippocampal neuron was isolated as previously reported (Bhargava et al. 2010). Briefly, $1 \sim 2$ day-old neonatal Sprague-Dawley rats were sacrificed and the whole brains were collected. The hippocampi were isolated from the neonatal brains and hippocampal neurons were harvested via collagenase digestion of hippocampus tissues. Subsequently, the hippocampal neurons were cultured as a monolayer at $37^{\circ} \mathrm{C}$ with a normoxic $95 \%$ air and $5 \% \mathrm{CO}_{2}$ incubator. The hippocampal neurons $\left(1 \times 10^{7}\right.$ cells/well $)$ were seeded in 24-well plates and transfected with $50 \mathrm{nM}$ miR-215 mimics (miR-215) or miR-215 negative control (miR-NC) after 3 days using Lipofectamine 2000 reagent. After transfection for $48 \mathrm{~h}$, the cells were treated with $20 \mu \mathrm{M}$ propofol for $0,2,4,6$ or $12 \mathrm{~h}$ under a normoxic 95\% air and 5\% $\mathrm{CO}_{2}$ incubator. Finally, the cells were examined in the following experiments.

\section{Real-time PCR}

Total RNA was isolated via TRIzol reagent (Invitrogen,Carlsbad,CA,USA). Synthesis of cDNA was conducted using TaqMan miRNA reverse transcription kit (Beyotime, Shanghai, China). The real-time PCR was carried out using SYBR Green (Takara, Dalian, China) on an ABI 7300 instrument. The miR-215 expression level was calculated via the $2^{-\Delta \Delta \mathrm{Ct}}$ method. The U6 was used as internal control. The primers used for this study were as follows: miR-215-F: AUGACCUAUGAAUUGACAGAC, miR-215-R: UCUGUCAUUUAGGCCAAUA. U6-F: GCTTCGCAGC ACATATACTAAAAT, U6-R: CGCTTCACGAATTT GCGTGTCAT.

\section{MTT assay}

A total of 3000 cells in $200 \mu \mathrm{l}$ of medium were plated into 96-well plates. The cells were incubated with 0.5 $\mathrm{mg} / \mathrm{ml} \quad 3$-(4,5-dimethyl-2-thiazolyl)-2,5-diphenyl-2-Htetrazolium bromide (MTT) solution (Beyotime, Shanghai, China) in $200 \mu \mathrm{l}$ of medium for $4 \mathrm{~h}$. The absorbance value was measured at a wavelength of $490 \mathrm{~nm}$.

\section{Apoptosis assay}

Cells $\left(10^{5}\right.$ cells $)$ were collected and suspended in Annexin $\mathrm{V}$ incubation solution. Then, the cells were stained with $5 \mu \mathrm{l}$ Annexin V-fluorescein isothiocyanate (FITC) and $5 \mu \mathrm{l}$ propidium iodide (PI) solution (Beyotime, Shanghai, China) in the dark for $20 \mathrm{~min}$. Apoptosis was then analyzed using flow cytometry.

\section{Oxidative stress and ROS measurement}

Transfected cells were treated with $20 \mu \mathrm{M}$ propofol for $6 \mathrm{~h}$ under a normoxic $95 \%$ air and $5 \% \mathrm{CO}_{2}$ incubator and then collected. Subsequently, the malondialdehyde (MDA), lactate dehydrogenase (LDH) and superoxide 
dismutase (SOD) were detected via ELISA kits (NJJC Bio Engineering Institute, Nanjing, China). The reactive oxygen species (ROS) level was examined in cells using $2^{\prime}$, $7^{\prime}$-dichlorofluorescin diacetate (DCFDA) for $30 \mathrm{~min}$ at $37^{\circ} \mathrm{C}$. The cells were observed, and data were analyzed through microplate reader.

\section{Dual luciferase reporter assay}

The wide-type 3'-UTR sequence of LATS2 contained the miR-215 binding site. Then, site-directed mutagenesis of the putative target site for miR-215 in wide-type 3'-UTR sequence of LATS2 was performed to generate the mutant-type 3 '-UTR sequence and the site-directed mutagenesis was showed in Fig. 3a. The primers designed by primer premier 5.0 was used to conduct amplification. The thermal cycle profile was as follows: denaturation for $20 \mathrm{~s}$ at $95^{\circ} \mathrm{C}$, annealing for $30 \mathrm{~s}$ at $54{ }^{\circ} \mathrm{C}$, and extension for $40 \mathrm{~s}$ at $72^{\circ} \mathrm{C}$. Subsequently, the sequences were inserted into the pmirGLO reporter vector (Promega, Shanghai, China) between XhoI and SalI restriction enzyme sites using T4 DNA Ligase to generate luciferase reporter constructs, and named by LATS2-WT and LATS2-MUT. Nucleotide sequences of the constructs were identified through DNA sequencing. HEK293T cells were cotransfected with $50 \mathrm{ng}$ of LATS2-WT or LATS2-MUT and $20 \mu \mathrm{M}$ of miR-215 or miR-NC for $48 \mathrm{~h}$. Finally, the luciferase activity was determined via dual luciferase reporter assay system (Promega, Shanghai, China).

\section{Western blot analysis}

Proteins were extracted via RIPA lysis buffer (Beyotime, Shanghai, China). The concentration was quantified by a BCA kit (Beyotime, Shanghai, China). Proteins $(45 \mu \mathrm{g})$ were separated by SDS-PAGE and transferred to polyvinylidene difluoride membrane (Millipore, USA). After blocking with 5\% non-fat milk for $1 \mathrm{~h}$ at $37^{\circ} \mathrm{C}$, the membrane was incubated with antibodies against LATS2 (1:1000, Abcam, Shanghai, China) and $\beta$-actin (1:1000, Abcam, Shanghai, China) overnight at $4{ }^{\circ} \mathrm{C}$. Subsequently, the secondary antibody (1:3000, Beyotime, Shanghai, China) was used to incubate the membrane for $2 \mathrm{~h}$ at $37^{\circ} \mathrm{C}$. The signals were visualized via an enhanced chemoluminescence kit (Takara, Dalian, China). The blots were analyzed with ImageJ software.

\section{Statistical analysis}

The data were presented as means \pm SD and analyzed with SPSS 18.0. Statistical analysis was conducted via two-tailed unpaired Student's t-test or one-way ANOVA with Tukey's test. $P$ value less than 0.05 was considered as statistically significant difference.

\section{Results}

Effect of miR-215 on propofol-induced apoptosis in neonatal rat hippocampal neuron

To explore the effect of miR-215 on propofol-induced apoptosis, we first examined miR-215 expression in neonatal rat hippocampal neuron. Real-time PCR showed that propofol treatment decreased miR-215 level in a time-dependent manner (Fig. 1a). MiR-215 level was increased in miR-215 mimics transfected neonatal rat hippocampal neuron treated with propofol (Fig. 1b). MTT assay demonstrated that propofol treatment reduced cell viability, whereas miR-215 mimics enhanced cell viability (Fig. 1c). In addition, apoptosis was increased by propofol treatment and miR-215 mimics suppressed propofolinduced apoptosis (Fig. 1d). These results indicate that miR-215 has a suppressive role in propofol-induced apoptosis in neonatal rat hippocampal neuron.

\section{Effect of miR-215 on propofol-induced oxidative stress in neonatal rat hippocampal neuron}

We then examined the role of miR-215 in propofolinduced oxidative stress. We first analyzed ROS level and the results showed that ROS generation was suppressed by miR-215 mimics in propofol-treated neonatal rat hippocampal neuron (Fig. 2a). MDA and LDH assays revealed that miR-215 mimics decreased MDA and LDH levels (Fig. 2b, c). On the other hand, miR-215 mimics increased SOD level (Fig. 2d). These findings suggest that miR-215 reduces propofol-induced oxidative stress in neonatal rat hippocampal neuron.

\section{LATS2 is a target of miR-215}

TargetScan analysis (http://www.targetscan.org/vert_72/) was used to predict the binding site of miR-215. The results showed that LATS2 is a target of miR-215 (Fig. 3a). To confirm the interaction between miR-215 and LATS2, dual luciferase reporter assay was performed and showed that miR-215 mimics decreased the relative luciferase activity of LATS2-WT. However, there was no effect on the relative luciferase activity in HEK293T cells co-transfected with miR-215 mimics and LATS2-MUT (Fig. 3b). Western blot analysis verified that miR-215 mimics inhibited LATS2 protein level in neonatal rat hippocampal neuron (Fig. 3c). The data indicated that LATS2 is a target of miR-215 and miR-215 represses LATS2 level.

\section{Downregulation of LATS2 induced by miR-215 overexpression in propofol-treated neonatal rat hippocampal neuron}

We then examined the effect of miR-215 overexpression on LATS2 in propofol-treated neonatal rat hippocampal neuron. Western blot analysis demonstrated that LATS2 protein level was elevated in neonatal rat 
A

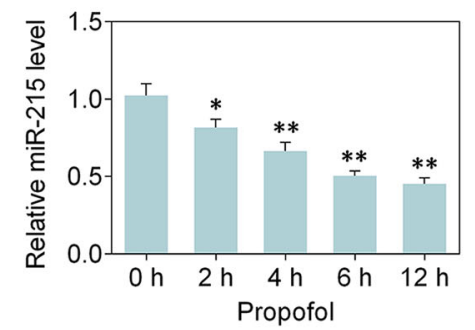

D

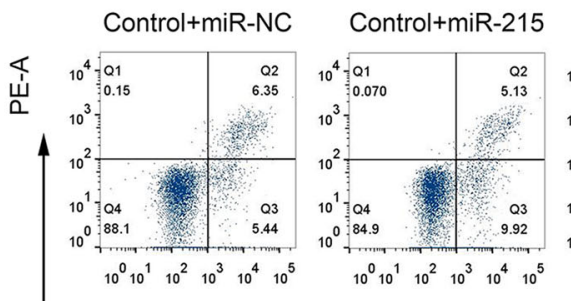

B

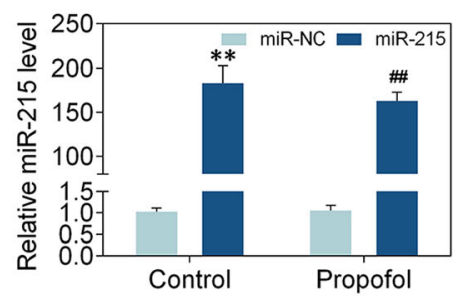

Propofol+miR-NC Propofol+miR-215

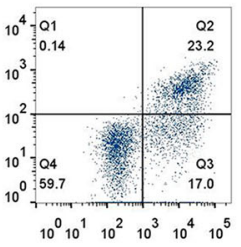

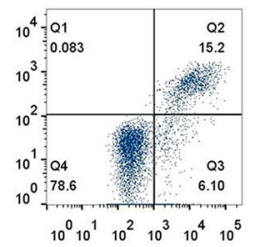

C
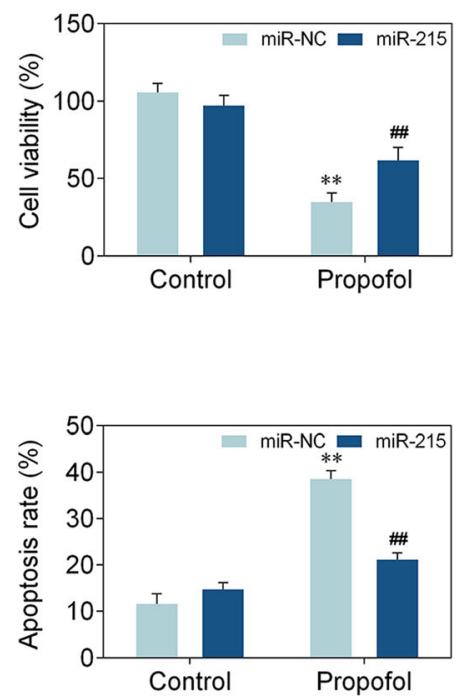

Fig. 1 Effect of miR-215 on propofol-induced apoptosis in neonatal rat hippocampal neuron. a miR-215 level was measured using real-time PCR. $n=3 .{ }^{*}, p<0.05 .{ }^{* *}, p<0.01$. ${ }^{*}$ vs $0 \mathrm{~h}$. Statistical analysis was conducted via one-way ANOVA by Tukey's test. $\mathbf{b}$ miR-215 level was detected via real-time PCR. Statistical analysis was conducted via one-way ANOVA by Tukey's test. c MTT assay was used to determine cell viability. Statistical analysis was conducted via one-way ANOVA by Tukey's test. $\mathbf{d}$ Flow cytometry analysis was used to detect apoptosis. Statistical analysis was conducted via one-way ANOVA by Tukey's test. $n=3 .{ }^{* *}, p<0.01 . \# \#, p<0.01 .{ }^{*}$ vs Control+NC mimics. \# vs Propofol+NC mimics

\section{A}

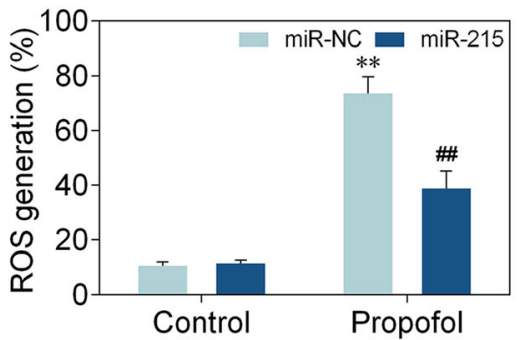

C

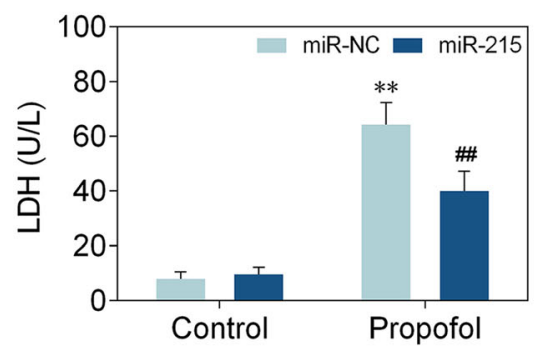

B

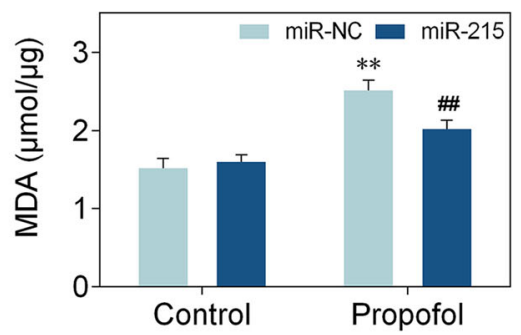

D

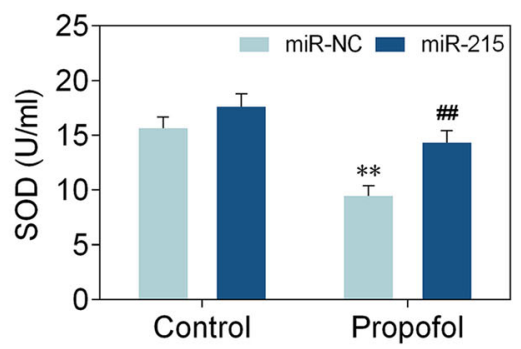

Fig. 2 Effect of miR-215 on propofol-induced oxidative stress in neonatal rat hippocampal neuron. a ROS level was examined in neonatal rat hippocampal neuron. Statistical analysis was conducted via one-way ANOVA by Tukey's test. b MDA detection was conducted in neonatal rat hippocampal neuron. Statistical analysis was conducted via one-way ANOVA by Tukey's test. c LDH assay examined the LDH level in neonatal rat hippocampal neuron. Statistical analysis was conducted via one-way ANOVA by Tukey's test. $\mathbf{d}$ SOD level from neonatal rat hippocampal neuron was assayed. Statistical analysis was conducted via one-way ANOVA by Tukey's test. $n=3 .{ }^{*}, p<0.01 . \# \#, p<0.01$. ${ }^{*}$ vs Control+NC mimics. \# vs Propofol+NC mimics 
A

LATS2-WT

miR-215

LATS2-MUT

$\mathrm{B}$

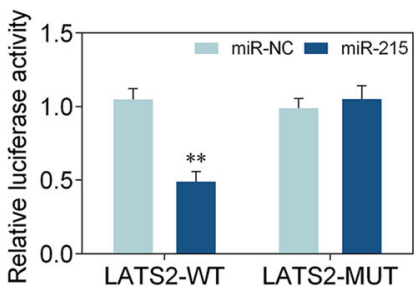

5'...acucgcugccucccAGGUCAg...3'

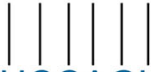

3' cagacaguuaaguaUCCAGUa 5'

5'...acucgcugccucccUCCAGUg...3'

Fig. 3 LATS2 is a target of miR-215. a TargetScan analysis was used to predict the binding site of miR-215. $\mathbf{b}$ Dual luciferase reporter assay confirmed the interaction between miR-215 and LATS2. Statistical analysis was conducted via one-way ANOVA by Tukey's test. c Western blot analysis detected LATS2 protein level in neonatal rat hippocampal neuron. Statistical analysis was carried out through two-tailed unpaired Student's t-test. $n=3 .{ }^{* *}, p<0.01$

hippocampal neuron with propofol treatment in a time-dependent manner (Fig. 4a). MiR-215 overexpression exhibited a suppressive role in propofolinduced increase of LATS2 level using western blot analysis (Fig. 4b). These results indicate that miR-215 overexpression downregulated LATS2 level in propofol-treated neonatal rat hippocampal neuron.

\section{MiR-215 attenuates propofol-induced apoptosis and oxidative stress in neonatal rat hippocampal neuron by targeting LATS2}

To test the hypothesis that miR-215 affects propofolinduced apoptosis and oxidative stress by targeting LATS2, we co-transfected miR-215 mimics and LATS2 in neonatal rat hippocampal neuron. Western blot analysis revealed that miR-215 mimics inhibited LATS2 protein level, whereas LATS2 overexpression elevated the protein level (Fig. 5a). Moreover, LATS2 overexpression suppressed the increase of cell viability induced by miR-215 mimics (Fig. 5b). Flow cytometry analysis showed that miR-215 mimics inhibited apoptosis, whereas LATS2 overexpression abrogated miR-215 induced inhibition of apoptosis (Fig. 5c). Furthermore, miR-215 mimics suppressed ROS, MDA and LDH levels, and promoted SOD level. On the other hand, LATS2 overexpression increased ROS, MDA and LDH levels, and decreased SOD level (Fig. 5d, e). These data suggest that miR-215 attenuates propofol-induced apoptosis and oxidative stress in neonatal rat hippocampal neuron by targeting LATS2.

\section{Discussion}

In this study, we found that miR-215 was decreased and LATS2 was increased in propofol-treated neonatal rat hippocampal neuron. Functional analysis showed that miR-215 has a suppressive role in propofol-induced apoptosis and oxidative stress in neonatal rat hippocampal neuron. Furthermore, we demonstrated that LATS2 is a target of miR-215, and miR-215 could reduce propofol-induced LATS2 level. LATS2 overexpression suppressed the effect of miR-215 on propofol-induced apoptosis and oxidative stress in neonatal rat hippocampal neuron. These data imply that miR-215 participates in the regulation of propofol-induced apoptosis and oxidative stress in neonatal rat hippocampal neuron by targeting LATS2.

Previous studies have shown that miRNAs are implicated in neurological diseases (Johnson et al. 2008; Asikainen et al. 2010; Hebert et al. 2008). Moreover, miRNAs dysregulation plays an important role in neurotoxicity (Kaur et al. 2012). Recently, miR-665 was found to be significantly increased in primary cultured astrocytes treated with propofol, and it inhibited BCL2L1 (Bcl-xl) (a suppressor of apoptosis) (Sun and Pei 2016). Moreover, miR-34a was discovered to be elevated after propofol treatment, and miR-34a knockdown could inhibit propofol-induced apoptosis (Li et al. 2018). Some miRNAs were reported to reverse the propofol-mediated effect. Wang et al. proved that miR-383 was downregulated by propofol treatment, and it could alter the propofol-induced upregulation of hippocampal neuron apoptosis (Wang et al. 2018a). Twaroski et al. found that 


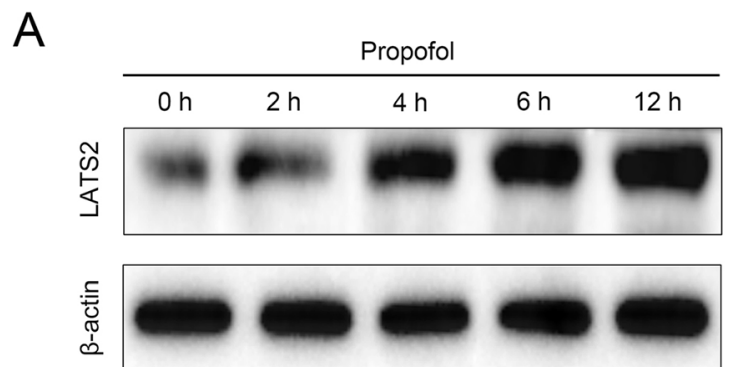

B

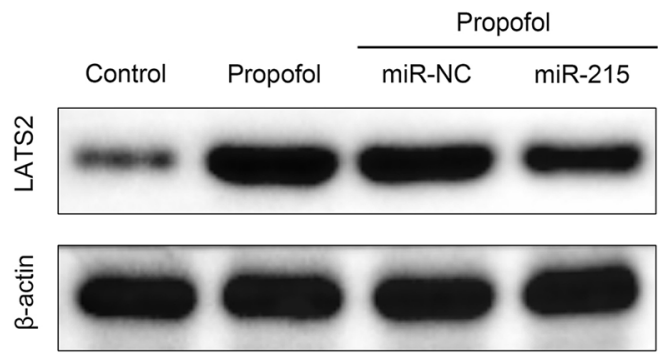

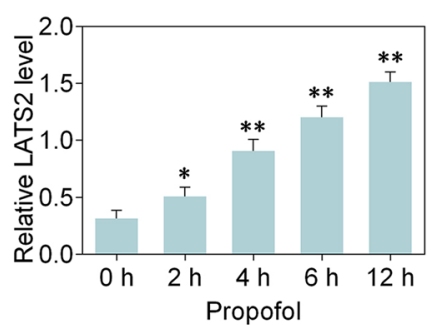

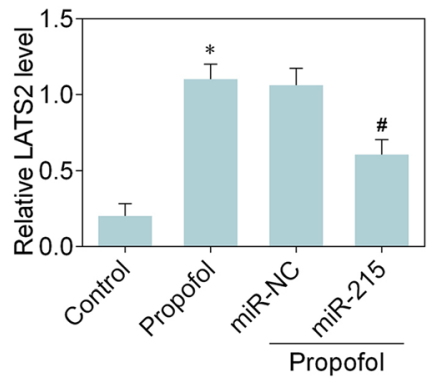

Fig. 4 Downregulation of LATS2 induced by miR-215 in propofol-treated neonatal rat hippocampal neuron. a LATS2 protein level was measured via western blot analysis in neonatal rat hippocampal neuron. Statistical analysis was conducted via one-way ANOVA by Tukey's test. $n=3 .{ }^{*}, p<$ 0.05. ${ }^{* *}, p<0.01 .{ }^{*}$ vs 0 h. b Western blot analysis detected LATS2 protein level in neonatal rat hippocampal neuron. Statistical analysis was conducted via one-way ANOVA by Tukey's test. $n=3 .{ }^{*}, p<0.05 . \#, p<0.05 .{ }^{*}$ vs Control. \# vs propofol+NC mimics

miR-21 was decreased in the neurons, and miR-21 overexpression alleviated propofol-caused cell death in human embryonic stem cells-derived neurons (Twaroski et al. 2014). Consistent with the later studies, our study revealed that miR-215 was downregulated in propofoltreated neonatal rat hippocampal neuron, and miR-215 mimics inhibited propofol-induced apoptosis. These results imply that miR-215 may act as a suppressive factor in propofol-induced apoptosis in neonatal rat hippocampal neuron.

Oxidative and antioxidative balance is disrupted upon oxidative stress. Oxidative and antioxidative products produced in cells include MDA, $\mathrm{LDH}$, and SOD (Rodrigo et al. 2016). It is reported that the balance between ROS production and scavenging is important in oxidative stress and has protective or damaging effects in several diseases (Aon et al. 2010). Oxidative stress and ROS have been found to be associated with a number of physiological and pathological processes (Huang et al. 2009). Increasing evidences have revealed that a large number of ROS could directly or indirectly induce oxidative damage to cells (Wang et al. 2018b; Lee et al. 2015). Notably, in our study, we discovered that miR-215 mimics reduced ROS, MDA and LDH levels, and increased SOD generation in propofol-treated neonatal rat hippocampal neuron, suggesting that miR-215 can negatively regulate propofol-induced oxidative stress in neonatal rat hippocampal neuron.

Accumulating evidences have shown that miRNAs carry out their functions by targeting mRNAs (Bartel 2009). Previous study proved that miR-410-3p has a neuroprotective effect on sevoflurane anesthesia-induced cognitive dysfunction by targeting C-X-C motif chemokine receptor 5 (Su et al. 2019). In addition, miR-133a$5 \mathrm{p}$ is involved in the protective effect of propofolmediated hepatic ischemia/reperfusion injury through targeting MAPK6 (Hao et al. 2017). MiR-665 was reported to participate in the neurotoxicity induced by propofol via targeting Bcl-2-like protein 1 BCL2L1 (Sun et al. 2015). Here we demonstrate that miR-215 could target LATS2. Moreover, the propofol-treated neonatal rat hippocampal neuron co-transfected with miR-215 mimics and LATS2 overexpression led to the suppression of miR-215 induced increase of cell viability and decrease of apoptosis and oxidative stress. The data indicate that miR-215 can attenuate propofol-induced apoptosis and oxidative stress in neonatal rat hippocampal neuron by targeting LATS2. However, to better clarify the role of miR-215 in neonatal rat hippocampal neuron by targeting LATS2, the function of miR-215 inhibition and LATS2 silencing in chemical-induced 


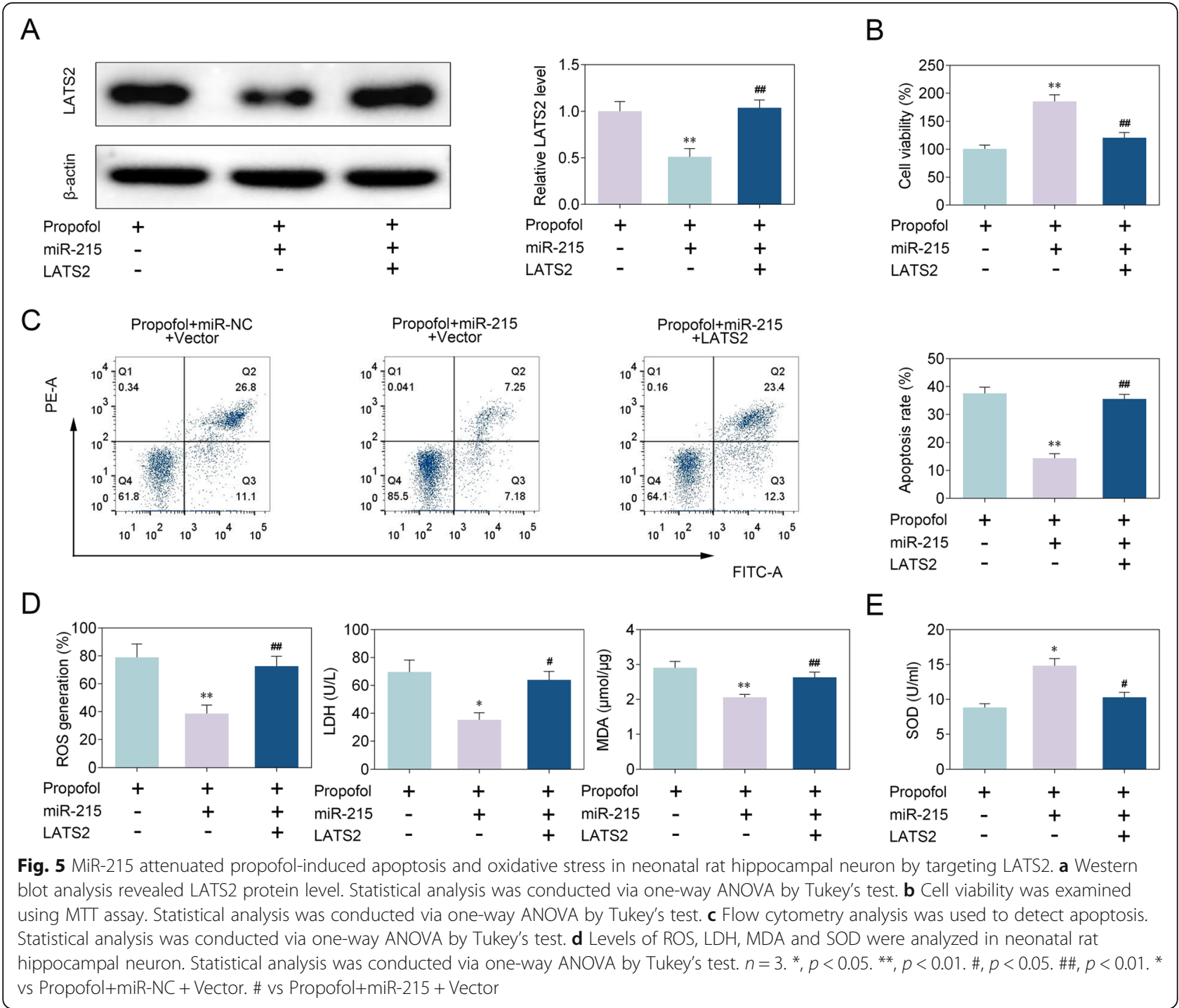

neonatal rat hippocampal neuron with high miR-215 expression will be performed in the near future.

\section{Conclusion}

In conclusion, in the current study we found that downregulation of miR-215 and upregulation of LATS2 were induced by propofol. Additionally, miR-215 overexpression alleviated propofol-induced apoptosis and oxidative stress in neonatal rat hippocampal neuron by targeting LATS2. Our results suggest miR-215 may provide a new therapeutic target to treat propofol-induced neuroapoptosis in developing neurons.

\section{Abbreviations}

miRNAs: MicroRNAs; LATS2: large tumor suppressor 2
Authors' contributions

FT and ZYL conceived and designed the experiments, LLZ, QY and TYL analyzed and interpreted the results of the experiments, HYG and QL performed the experiments. The author(s) read and approved the final manuscript

\section{Funding}

Not applicable.

\section{Availability of data and materials}

All data generated or analyzed during this study are included in this published article.

\section{Ethics approval and consent to participate}

The cell isolation procedures in this study were approved by Guide for the Care and Use of Laboratory Animals and in agreement with the Ethics Committee of The first affiliated hospital of Nanchang University.

\section{Consent for publication}

Not Applicable.

\section{Competing interests}

The authors state that there are no conflicts of interest to disclose. 
Received: 7 January 2020 Accepted: 23 April 2020

Published online: 06 May 2020

\section{References}

Aon MA, Cortassa S, O'Rourke B. Redox-optimized ROS balance: a unifying hypothesis. Biochim Biophys Acta. 2010;1797:865-77.

Asikainen S, Rudgalvyte M, Heikkinen L, Louhiranta K, Lakso M, Wong G, et al. Global microRNA expression profiling of Caenorhabditis elegans Parkinson's disease models. J Mol Neurosci. 2010:41:210-8.

Aylon Y, Michael D, Shmueli A, Yabuta N, Nojima H, Oren M. A positive feedback loop between the p53 and Lats2 tumor suppressors prevents tetraploidization. Genes Dev. 2006;20:2687-700.

Aylon Y, Ofir-Rosenfeld Y, Yabuta N, Lapi E, Nojima H, Lu X, et al. The Lats2 tumor suppressor augments $\mathrm{p53}$-mediated apoptosis by promoting the nuclear proapoptotic function of ASPP1. Genes Dev. 2010;24:2420-9.

Bartel DP. MicroRNAs: target recognition and regulatory functions. Cell. 2009;136: 215-33.

Bhargava N, Das M, Edwards D, Stancescu M, Kang JF, Hickman JJ. Coexpression of glutamate vesicular transporter (VGLUT1) and choline acetyltransferase (ChAT) proteins in fetal rat hippocampal neurons in culture. In Vitro Cell Dev Biol Anim. 2010;46:685-92.

Bosnjak ZJ, Logan S, Liu Y, Bai X. Recent insights into molecular mechanisms of Propofol-induced developmental neurotoxicity: implications for the protective strategies. Anesth Analg. 2016;123:1286-96.

Brandt ZJ, North PN, Link BA. Somatic mutations of lats2 cause peripheral nerve sheath tumors in Zebrafish. Cells. 2019:8:972.

Chidambaran V, Costandi A, D'Mello A. Propofol: a review of its role in pediatric anesthesia and sedation. CNS Drugs. 2015;29:543-63.

Cui Y, Ling-Shan G, Yi L, Xing-Qi W, Xue-Mei Z, Xiao-Xing Y. Repeated administration of propofol upregulated the expression of c-Fos and cleavedcaspase-3 proteins in the developing mouse brain. Indian J Pharmacol. 2011; 43:648-51.

Fabian MR, Sonenberg N, Filipowicz W. Regulation of mRNA translation and stability by microRNAs. Annu Rev Biochem. 2010;79:351-79.

Hao W, Zhao ZH, Meng QT, Tie ME, Lei SQ, Xia ZY. Propofol protects against hepatic ischemia/reperfusion injury via miR-133a-5p regulating the expression of MAPK6. Cell Biol Int. 2017:41:495-504.

Hebert SS, Horre K, Nicolai L, Papadopoulou AS, Mandemakers W, Silahtaroglu AN, et al. Loss of microRNA cluster miR-29a/b-1 in sporadic Alzheimer's disease correlates with increased BACE1/beta-secretase expression. Proc Natl Acad Sci U S A. 2008;105:6415-20.

Huang Q, Wu YT, Tan HL, Ong CN, Shen HM. A novel function of poly(ADPribose) polymerase-1 in modulation of autophagy and necrosis under oxidative stress. Cell Death Differ. 2009;16:264-77.

Ji Y, Wang D, Zhang B, Lu H. MiR-361-3p inhibits beta-amyloid accumulation and attenuates cognitive deficits through targeting BACE1 in Alzheimer's disease. J Integr Neurosci. 2019;18:285-91.

Jiang $Q$, Wang $Y$, Shi X. Propofol inhibits neurogenesis of rat neural stem cells by Upregulating MicroRNA-141-3p. Stem Cells Dev. 2017;26:189-96.

Johnson R, Zuccato C, Belyaev ND, Guest DJ, Cattaneo E, Buckley NJ. A microRNA-based gene dysregulation pathway in Huntington's disease. Neurobiol Dis. 2008:29:438-45.

Kaur P, Armugam A, Jeyaseelan K. MicroRNAs in neurotoxicity. J Toxicol. 2012; 2012:870150

Ke H, Pei J, Ni Z, Xia H, Qi H, Woods T, et al. Putative tumor suppressor Lats2 induces apoptosis through downregulation of $\mathrm{BCl}-2$ and $\mathrm{BCl}-x(\mathrm{~L})$. Exp Cell Res. 2004;298:329-38.

Lee YM, Song BC, Yeum KJ. Impact of volatile anesthetics on oxidative stress and inflammation. Biomed Res Int. 2015:2015:242709.

Li GF, Li ZB, Zhuang SJ, Li GC. Inhibition of microRNA-34a protects against propofol anesthesia-induced neurotoxicity and cognitive dysfunction via the MAPK/ERK signaling pathway. Neurosci Lett. 2018;675:152-9.

Rodrigo S, Rodriguez L, Otero P, Panadero MI, Garcia A, Barbas C, et al. Fructose during pregnancy provokes fetal oxidative stress: the key role of the placental heme oxygenase-1. Mol Nutr Food Res. 2016;60:2700-11.

Shukla GC, Singh J, Barik S. MicroRNAs: processing, maturation, target recognition and regulatory functions. Mol Cell Pharmacol. 2011;3:83-92.

Su R, Sun P, Zhang D, Xiao W, Feng C, Zhong L. Neuroprotective effect of miR410-3p against sevoflurane anesthesia-induced cognitive dysfunction in rats through PI3K/Akt signaling pathway via targeting C-X-C motif chemokine receptor 5. Genes Genomics. 2019;41:1223-31.
Sun H, Zhong D, Jin J, Liu Q, Wang H, Li G. Upregulation of miR-215 exerts neuroprotection effects against ischemic injury via negative regulation of Act1/LL-17RA signaling. Neurosci Lett. 2018;662:233-41.

Sun WC, Liang ZD, Pei L. Propofol-induced rno-miR-665 targets BCL2L1 and influences apoptosis in rodent developing hippocampal astrocytes. Neurotoxicology. 2015;51:87-95.

Sun WC, Pei L. Rno-miR-665 targets BCL2L1 (BCL-xl) and increases vulnerability to propofol in developing astrocytes. J Neurochem. 2016;138:233-42.

Twaroski DM, Yan Y, Olson JM, Bosnjak ZJ, Bai X. Down-regulation of microRNA21 is involved in the propofol-induced neurotoxicity observed in human stem cell-derived neurons. Anesthesiology. 2014;121:786-800.

Unoki M, Nakamura Y. Growth-suppressive effects of BPOZ and EGR2, two genes involved in the PTEN signaling pathway. Oncogene. 2001;20:4457-65.

Wang X, Ding G, Lai W, Liu S, Shuai J. MicroRNA-383 upregulation protects against propofol-induced hippocampal neuron apoptosis and cognitive impairment. Exper Therapeutic Med. 2018a;15:3181-8.

Wang Y, Branicky R, Noe A, Hekimi S. Superoxide dismutases: dual roles in controlling ROS damage and regulating ROS signaling. J Cell Biol. 2018b;217: 1915-28.

Wei H. The role of calcium dysregulation in anesthetic-mediated neurotoxicity. Anesth Analg. 2011;113:972-4.

Yabuta N, Fujii T, Copeland NG, Gilbert DJ, Jenkins NA, Nishiquchi H, et al. Structure, expression, and chromosome mapping of LATS2, a mammalian homologue of the Drosophila tumor suppressor gene LATS/warts. Genomics. 2000;63:263-70.

Zhang H, Deo M, Thompson RC, Uhler MD, Turner DL. Negative regulation of yap during neuronal differentiation. Dev Biol. 2012;361:103-15.

Zhang S, Liang Z, Sun W, Pei L. Repeated propofol anesthesia induced downregulation of hippocampal miR-132 and learning and memory impairment of rats. Brain Res. 2017;1670:156-64.

Zheng X, Huang H, Liu J, Li M, Liu M, Luo T. Propofol attenuates inflammatory response in LPS-activated microglia by regulating the miR-155/SOCS1 pathway. Inflammation. 2018:41:11-9.

\section{Publisher's Note}

Springer Nature remains neutral with regard to jurisdictional claims in published maps and institutional affiliations.

Ready to submit your research? Choose BMC and benefit from:

- fast, convenient online submission

- thorough peer review by experienced researchers in your field

- rapid publication on acceptance

- support for research data, including large and complex data types

- gold Open Access which fosters wider collaboration and increased citations

- maximum visibility for your research: over $100 \mathrm{M}$ website views per year

At $\mathrm{BMC}$, research is always in progress.

Learn more biomedcentral.com/submissions 